\title{
2D/3D Hybrid Character Animation on "Spirit"
}

\author{
Doug Cooper, Digital Supervisor \\ DreamWorks Feature Animation \\ coop@anim.dreamworks.com
}

\begin{abstract}
DreamWorks Feature animated film "Spirit" takes new steps in the hybridization of $2 \mathrm{D}$ and $3 \mathrm{D}$ production techniques for Character Animation, even to the extent of using both approaches within the same shot.
\end{abstract}

\section{Introduction}

The primary character in "Spirit" is a traditionally drawn 2D animated horse, which inhabits the richly detailed tapestry of the American Wild West at the turn of the century. The heavy use of $3 \mathrm{D}$ sets and camera moves brought an extra challenge to the traditional animators in needing to precisely match the sometimes subtle and slow perspective changes that occurred throughout a shot.

\section{3D Reference}

To help traditional animators meet this challenge, we took animated cycles of a 3D version of the character, and printed them onto animation paper. The traditional animators used these prints as perspective, volume, and scale reference when making their drawings. The animators did not rotoscope the CG renders, but instead used it solely for scale and perspective reference.

\subsection{Digital Stunt Doubles}

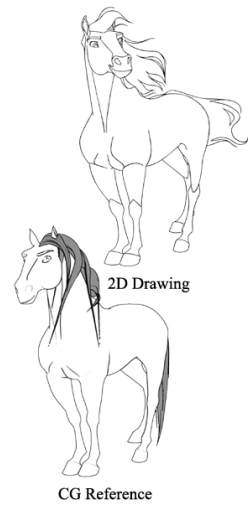

We modeled and setup full 3D versions of the main characters in the film to use as "digital stunt doubles" for medium to long shots, and in shots with very complicated 3D camera moves. For closeup performances, we still used the traditionally animated characters so we could accomplish the highest quality of animation performance.

\subsection{Take-overs}

In several long and complicated shots, we used both 2D and 3D techniques within the same shot simultaneously to achieve a costs savings over animating the entire shot traditionally.

For example, in one elaborate scene, we begin with a tracking camera move following Spirit with a herd of horses. Spirit is fully $3 \mathrm{D}$, as well as all the horses in the herd, and the environment. As the camera pans down and trucks into a close-up of Spirit, we "take-over" to a completely traditionally drawn version of Spirit in the middle of the shot. Spirit remains traditionally drawn for the entire close-up camera circle around him. As the camera trucks back to reveal the expansive 3D landscape, we again takeover to a fully $3 \mathrm{D}$ version of the character. Using this technique allowed us to achieve significant cost savings of several hundred drawings.

\subsubsection{From 3D to 2D}

We started first by animating the entire shot in $\mathrm{CG}$ - portions that we planned to draw were not animated fully since they were only needed for reference.

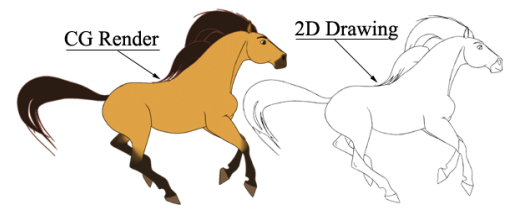

The CG animation was then rendered with a toon shader, and printed onto animation paper. The traditional animators then precisely matched several frames of the $\mathrm{CG}$ as drawings before taking over fully and animating the close-up. When it was time to transition back to $3 \mathrm{D}$, the animators would again match the $\mathrm{CG}$ for a few frames. After the transition back to 3D, we kept a traditionally animated mane and tail composited onto the character for stylistic reasons.

\subsubsection{Matching CG lines to 2D lines}

After the drawings were completed and scanned in, we adjusted the toon shader parameters for the CG to more precisely match the line thickness of the scanned drawings. A simple cross-dissolve was used for the transition. We chose the transition time from 3D to $2 \mathrm{D}$ carefully to correspond with the character coming to an abrupt stop on a hill in the shot, helping to disguise the transition. The transition back to $3 \mathrm{D}$ was harder, because the movement was much slower.

\subsubsection{Matching drawn lighting to CG lighting}

On a 2D animated film, the lighting that appears on 2D drawn characters is accomplished by drawn "tone mattes" which define the areas of light and shadow. Compositors use the mattes to darken or lighten the character's colors as desired. Traditionally drawn tone mattes are highly subjective in their design, as opposed to accurately reflecting the $3 \mathrm{D}$ volume of the character.

Matching these designs with 3D lighting proved to be very difficult. Whenever possible, we rendered the 3D lighting first, and asked the traditional Effects animators to match their designs to that for the transition frames. When the match wasn't accurate enough, we used roto-mattes generated in compositing to fix the $3 \mathrm{D}$ renders, or the $2 \mathrm{D}$ drawings to bring them closer together.

\section{Conclusions and Future Work}

"Spirit" further blurs the line between animation techniques towards a future were qualifiers such as "digital" or "traditional" will be invalid, and we'll simply have to call it "animation". On our upcoming film "Sinbad", we have expanded the use of Digital Characters, 3D sets and Effects, and moved to an entirely 3D layout process. We're also developing more digital 2D tools for drawing shadows and tones directly in the computer, and training all of our traditional animators in $3 \mathrm{D}$ animation tools so they can apply their performance talents in the appropriate medium when needed. 